\title{
Fast Extragalactic X-ray Transients From Binary Neutron Star Mergers
}

\author{
Shlomo Dado and Arnon Dar \\ Physics Department, Technion, Haifa, Israel
}

\begin{abstract}
The observed light curves and other properties of the two extragalactic fast x-ray transients, CDF-S XT1 and CDF-S XT2, which were discovered recently in archival data of the Chandra Deep Field-South (CDF-S) observations, indicate that they belong to two different populations of X-ray transients. XT1 seems to be an x-ray flash (XRF), i.e., a narrowly beamed long duration gamma ray burst viewed from far off-axis while XT2 seems to be a nebular emission powered by a newly born millisecond pulsar in a neutron stars binary merger.
\end{abstract}




\section{INTRODUCTION}

The production of gamma ray bursts (GRBs) in neutron stars binary (NSB) mergers due to gravitational wave (GW) emission was first suggested in 1984 [1] to be due to explosion (later called a kilonova [2]) of the lighter neutron star in NSB mergers following tidal mass loss. Later in 1987, it was suggested that GRBs are produced by neutrino annihilation fireballs [3] formed around the newly born neutron star remnant in NSB mergers driven by gravitational wave (GW) emission and in stripped envelope supernova explosions. But, the observations of GRBs by the Compton Gamma-Ray Observatory (CGRO), soon after its launch in 1991, indicated that neither one of these two mechanisms was powerful enough to produce observable GRBs at the very large cosmological distances [4] indicated by the CGRO observations. Thus, in 1994, the fireball mechanism [5] for production of prompt emission in GRBs was replaced by inverse Compton scattering (ICS) of external light by a highly relativistic bipolar jets of plasmoids of ordinary plasma, launched by fall back ejecta on the remnants of NSB mergers and stripped envelope supernova explosions and in the collapse of neutron stars to quark stars or black holes due to mass accretion in high mass X-ray binaries [6]. Shortly after the discovery in 1997 by the Italian-Dutch BeppoSAX satellite [7] discovered that GRBs are followed by a long-lived x-ray afterglow, and the following discoveries of their afterglows at longer wave lengths [8], the jet model of GRBs [6] was extended to predict also the properties of GRB afterglows [9]. The spherical fireball model, however, which predicted the existence of GRB afterglows with a single power light curves [10], was later replaced, first by conical $e^{+} e^{-} \gamma$ fireballs [11], and finally by conical jets of ordinary plasma [12].

The first NSB merger detected in gravitational waves by the Ligo-Virgo detectors [13], GW170817, was followed $1.74 \pm 0.5 \mathrm{~s}$ later by a short duration gamma ray burst GRB170817A [14]. Moreover, very large base interferometry (VLBI) late time follow up observations of the radio afterglow of GRB170817A provided the first direct observational evidence [15] that NSB mergers launch narrowly collimated jets of "superluminal" plasmoids which produce narrowly beamed short gamma ray burst (SGRB) pulses with narrowly beamed late time afterglow [15]. However, its early-time optical afterglow was claimed to be produced by a kilonova [16], or a nebular emission with a thermalized bremsstrahlung, powered by a 
millisecond pulsar (MSP) remnant of the NSB merger [17]. The claimed kilonova, however, had an early time bolometric light curve much different from that claimed before to be associated with SGRB [18], while its bolometric light curve displayed the same "universal shape" [19] as that of about half of the well sampled early time x-ray afterglows of ordinary (viewed near axis) SGRBs, which were measured before with the Swift XRT [20].

GW170817/GRB170817A was the first indisputable evidence that NSB mergers produce SGRBs with a narrowly beamed late time afterglow, and perhaps an early time isotropic afterglow emission. By chance, two days before this event, it was predicted [20] that the visible smoking guns of NSB mergers are mostly millisecond pulsars remnants whose spin down powers an isotropic nebular emission with nearly a universal shape light curve [21].

Recently, two extragalactic fast x-ray transients, CDF-S XT1 [22] associated with a faint galaxy at a photometric redshift $z_{p h} \sim 2.2$ and CDF-S XT2 associated with a galaxy at redshift $z=0.738$ [23] were discovered in archival data of the Chandra Deep Field-South (CDF-S) observations. It was suggested that both are X-ray nebular emission powered by the spin down of newly born magnetars in NSB mergers, which produced SGRBs that are beamed away from Earth $[23,24]$. In this paper, we provide supportive evidence that CDF-S XT2 was an early time isotropic orphan x-ray afterglow of NSB merger which produced a remnant MSP. However, the light curve of CDF-XT1 indicates that, more likely, it was an X-ray flash (XRF) i.e., a prompt emission pulse of a narrowly beamed long duration gamma ray burst (LGRB) viewed from far off axis [38], rather than a nebular emission powered by the spin down of a newly born MSP [26].

\section{ORPHAN X-RAY AFTERGLOWS OF GRBS}

There is mounting observational data indicating that LGRBs and SGRBs are narrowly beamed along the direction of motion of highly relativistic jets of plasmoids which produce them [27]. Distant observers located outside the beaming cone of such jets miss their prompt gamma ray emission and their beamed afterglows. However, the deceleration of jets in the circumburst medium decreases their bulk motion Lorenz factor $\gamma(t)$ and widens their beaming cone of their radiation. Once their beaming cone includes the distant observer, their afterglow radiations become visible. However, so far, no such orphan GRB afterglows [28] have been detected. That could have been because of various reasons, such as lack of 
a unique signature, a luminosity below detection threshold by the time their beaming cone has expanded enough to include Earth, a very small sky coverage in very deep searches, and a small signal to background ratio.

Another type of orphan electromagnetic afterglows, which can be seen from large cosmic distances, is an isotropic early time nebular emission powered by a newly born MSP [21]. Such MSPs can be the remnants of NSB mergers which do not not produce a GRB, or which produce narrowly beamed GRBs that do not point to Earth. In both cases they can produce an x-ray emission with a "universal" shape [19]. Indeed, a large fraction of SGRBs which point to Earth and have a well sampled early time x-ray afterglow light curve have a universal shape expected from a nebular emission powered by a newly born MSP [19]. Such afterglows can be seen up to very large cosmic distances. Moreover, if the birth of an MSP is accompanied by a narrowly beamed SGRB which points away from Earth, then the x-ray nebular emission appears as an orphan extragalactic fast x-ray transient [21].

Below, we show first that the extragalactic fast X-ray transient CDF-S XT2 discovered in the Chandra Deep Field-South observations $[22,23]$ and a large fraction of the well sampled early time X-ray afterglow of SGRBs [19] share the same "universal shape" light curves [19] powered by newly born MSPs [29]. Next we show that the estimated full sky rate of CDF-S XT2 like events [24] is consistent with that expected from the local cosmic rate of neutron star mergers [30]. Together, they provide supporting evidence to the suggestion that CDF-S XT2 [23] probably is the early time nebular emission powered by the spin down of a newly born pulsar of a far off axis SGRB produced in NSB merger [21,23,24,25].

\section{NEBULAR EMISSION POWERED BY NEWLY BORN MSPS}

The spin down energy of a pulsar with a constant moment of inertia $I$, is given by

$$
\dot{E}=4 \pi^{2} \nu \dot{\nu} I
$$

where $\nu$ is its spin frequency. For a pulsar with braking index $n$ defined by

$$
\dot{\nu}=-k \nu^{n}
$$

where $k$ is a time independent constant, the rate of its rotational energy loss is given by

$$
\dot{E}(t)=\dot{E}(0)\left(1+t / t_{b}\right)^{-(n+1) /(n-1)},
$$


with

$$
t_{b}=-\nu(0) /(n-1) \dot{\nu}(0)=P(0) /(n-1) \dot{P}(0),
$$

where $P=1 / \nu$ is the pulsar's period.

For a spin down dominated by the emission of magnetic dipole radiation (MDR) in vacuum $n=3$ and

$$
L(t)=L(0) /\left(1+t / t_{b}\right)^{2}
$$

where $L(t)=\dot{E}$. As long as the early time x-ray afterglows of SGRBs from NSB mergers are pulsar wind nebula (PWN) emission powered by a constant fraction $\eta$ of the spin down energy of a newly born pulsar with a braking index $n=3$, the early time x-ray afterglow of both a visible and an invisible SGRBs have the universal behavior,

$$
F_{x}(t) / F_{x}(0)=\left[1+t_{s}\right]^{-2}
$$

where $F_{x}(t)$ is the measured energy flux of the X-ray afterglow of the SGRB and $t_{s}=t / t_{b}$.

In Figure 1 the reported x-ray light curve of CDF-S XT2 [23], reduced to the dimensionless universal form given by Eq.(6), is compared to the early time light curves of the x-ray afterglow of about half of the SGRBs with a well sampled x-ray afterglow measured with the Swift XRT and reported in the Swift-XRT Light Curves Repository [20]. For each SGRB afterglow the values of the parameters $F_{x}(0)$ and $t_{b}$ were obtained from a best fit of Eq.(6) to the the measured light curves. Their values were reported in Table I of [19]. A best fit of Eq.(6) to the 0.3-10 keV X-ray light curve of CDF-S XT2 [23] has yielded the best fit values, $F_{x}(0)=8.8 \times 10^{-13} \mathrm{erg} / \mathrm{cm}^{2} \mathrm{~s}$ and $t_{b}=1705 \mathrm{~s}$.

\section{LOWER BOUND ON MSP MAGNETIC FIELD}

If the spin down of the newly born pulsar is dominated by magnetic dipole radiation, the magnetic field $B_{p}$ at the pulsar's magnetic poles satisfies [31]

$$
B \sin \alpha \approx 6.8 \times 10^{19}[P \dot{P}]^{1 / 2} \text { Gauss }
$$

where $P$ is in seconds and $\alpha$ is the angle of the magnetic poles relative to the rotation axis.

The initial period of the pulsar could be estimated [19] from the best fit parameters $F_{x}(0)$, $t_{b}$ and the luminosity distance of the PWN only when the fraction $\eta$ of entire spin down energy of the pulsar, which has been converted by the PWN to the observed afterglow of 


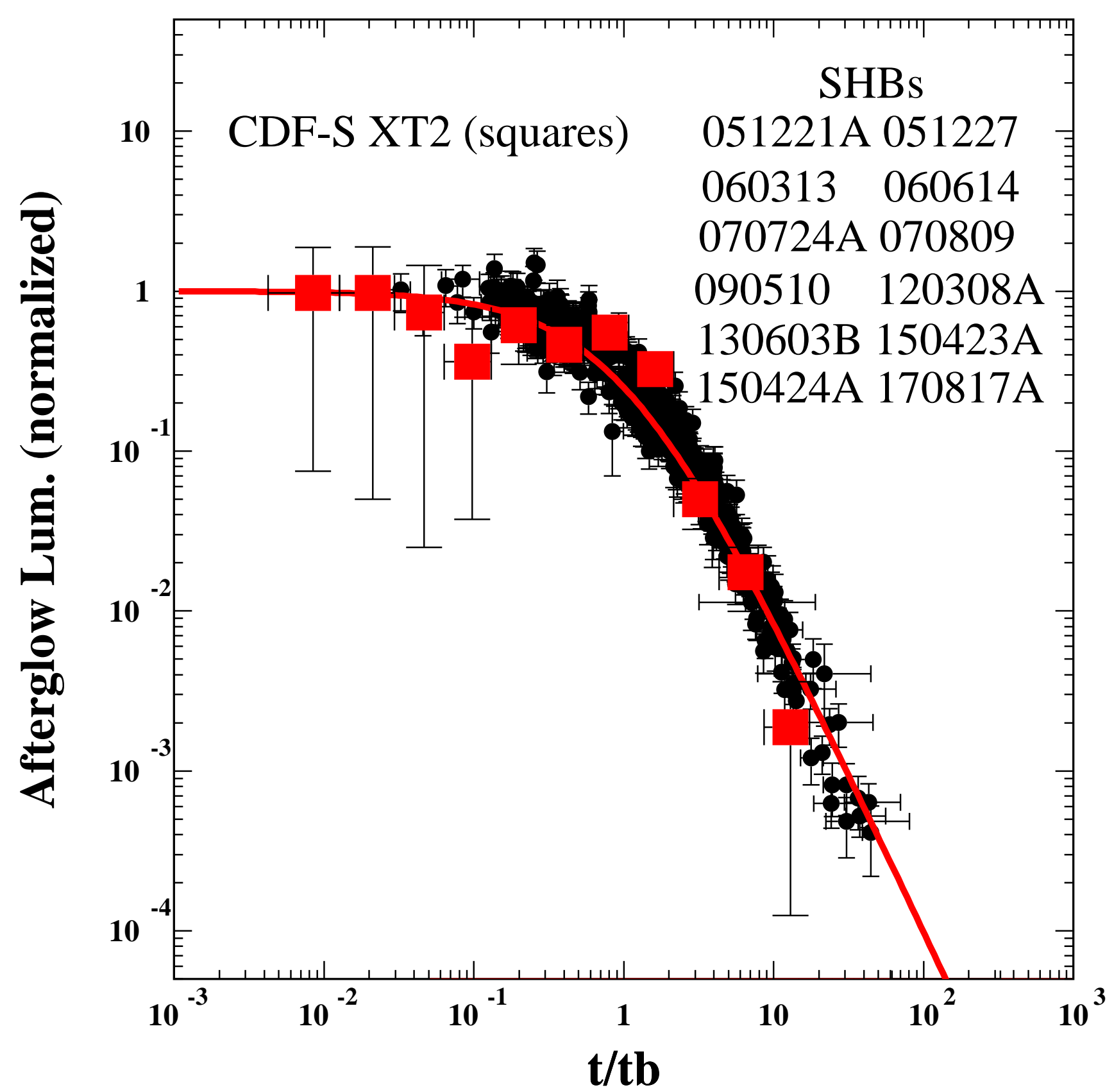

FIG. 1: Comparison between the scaled 0.3-10 keV light curves of the well sampled X-ray afterglow of SGRBs during the first couple of days after burst measured with the Swift XRT [20] and the 0.3-10 keV light curve of CDF-S XT2 [23]. The line is the expected universal behavior given by Eq.(6) of a PWN afterglow powered by a newly born millisecond pulsar with a braking index $n=3$. 
the SGRB, is known. However, usually the exact geometry of the PWN and the fraction of the pulsar spin energy converted to X-ray emission in the PWN are not known. As a result the value of $\eta$ is usually unknown. Moreover there is no reliable evidence that millisecond pulsars spin down by the emission of magnetic dipole radiation. That, and the lack of reliable evidence that MSPs spin down by magnetic dipole radiation [32] prevents the use of Eq.(7) to obtain a reliable estimate of the magnetic field of the neutron star at the magnetic poles.

However, if the widespread assumption that MSPs spin down mainly by magnetic dipole radiation is correct, then a lower bound on the magnetic field at the poles can be estimated from the best fit value of $t_{b}$ obtained from the best fit of Eq.(6) to the early X-ray afterglow of SGRBs powered by newly born pulsars, as follows. Substitution of the lower classical limit $P \geq 2 \pi R / c \approx 0.2 \mathrm{~ms}$ for a canonical pulsar with a radius $R \approx 10 \mathrm{~km}$ and a surface velocity equal to the speed of light, and substituting it in Eq. (6), and the use of the relation $\dot{P}(0)=P(0) / 2 t_{b}$ valid for a braking index $n=3$, which is valid for a constant magnetic field in vacuum, imply the lower limit,

$$
B_{p}(0) \gtrsim 10^{16} \sqrt{(1+z) /\left(t_{b} / s\right)} \text { Gauss. }
$$

Eq.(8) yields $B_{p}(0) \gtrsim 3 \times 10^{14}$ Gauss for CDF-S XT2 at its redshift $z=0.735$ [23].

The best fit of eq.(6) to the early time optical afterglow [33] of GRB170817A at $z=0.0093$, has yielded $t_{b} \approx 117374 \mathrm{~s}$, [19]. Hence, eq.(8) yields $B_{p}(0) \gtrsim 10^{14}$ Gauss for a pulsar powering the early time bolometric light curve of the optical afterglow of GRB170817A.

\section{THE FULL SKY RATE OF ORPHAN MSP POWERED AFTERGLOWS}

If the cosmic rate of NSB mergers in a comoving volume as a function of redshift $z$ is proportional to the star formation rate, SFR(z), e.g., if they are produced mainly by fission of a fast rotating core in core collapse supernova explosions of massive stars [17], then the production rate of pulsar powered afterglows by NSB mergers in a comoving volume is given by $[34]$

$$
\frac{d \dot{N}}{d z} \propto \operatorname{SFR}(z) \frac{d V_{c}(z)}{d z} \frac{1}{1+z}
$$


where $V_{c}(z)$ is the comoving volume at redshift $z$. In a standard $\Lambda$ CDM cosmology, $d V_{c}(z) / d z$ is given by

$$
\frac{d V_{c}(z)}{d z}=\frac{c}{H_{0}} \frac{4 \pi\left[D_{c}(z)\right]^{2}}{\sqrt{(1+z)^{3} \Omega_{M}+\Omega_{\Lambda}}},
$$

where $H_{0}$ is the current Hubble constant, $\Omega_{M}$ and $\Omega_{\Lambda}$ are, respectively, the current density of ordinary energy and of dark energy, in critical energy-density units, and $D_{c}(z)$ is the comoving distance at a red shift $z$, which satisfies

$$
D_{c}(z)=\frac{c}{H_{0}} \int_{0}^{z} \frac{d z^{\prime}}{\sqrt{\left(1+z^{\prime}\right)^{3} \Omega_{M}+\Omega_{\Lambda}}} .
$$

In order to estimate the full sky rate of NSB mergers, $\dot{N}(z)$, as given by Eqs.(9-11) we have adopted the current best values of the cosmological parameters obtained from the combined WMAP and Planck data [35]: a Hubble constant $H_{0}=67.4 \mathrm{~km} / \mathrm{s} \mathrm{Mpc,} \Omega_{M}=$ 0.315 and $\Omega_{\Lambda}=0.685$ and the $\operatorname{SFR}(\mathrm{z})$ compiled and standardized in [36] and [37] from optical measurements. This standardized SFR(z) is well approximated [33] by a log-normal distribution,

$$
\operatorname{SFR}(z) \approx 0.25 e^{-[l n((1+z) / 3.16)]^{2} / 0.524} \mathrm{M}_{\odot} \mathrm{Mpc}^{-3} \mathrm{y}^{-1} .
$$

Assuming that the cosmic rate of neutron star mergers (NSMs) as a function of redshift is proportional to SFR(z) given by Eq.(12), and that the rate of NSMs in a comoving volume of $\mathrm{Gpc}^{3}$ is $(1540+3200 /-1200) G_{p c}^{-3} y^{-1}$, as estimated in [13] from the Ligo-Virgo GW

observations, then the expected full sky rate $\dot{N}(\leq z)$ of NSMs in the standard cosmological model with the updated values of the cosmological parameters measured with Planck [34], is shown in Figure 2. This rate, to a good approximation, is also the expected rate of orphan early time afterglows produced by the majority of SGRBs which point away from Earth. Their full sky rate obtained from their estimated rate $59+77 /-38$ evt $\mathrm{y}^{-1} \mathrm{deg}^{-2}$ in [24] from the CDF-S observations of XT1 and XT2 [24], after subtracting the contribution of $\mathrm{XT1}$, is also indicated in Figure 2.

\section{CDF-S XT1 AS FAR OFF-AXIS LGRB}

In the cannonball model of GRBs, the predicted pulse shape above a minimal energy $E_{m}$ of prompt emission pulses of GRBs has the behavior [26,38],

$$
\frac{d N_{\gamma}\left(E>E_{m}\right)}{d t} \propto \frac{t^{2} \exp \left[-E_{m} / E_{p}(t)\right]}{\left(t^{2}+\Delta^{2}\right)^{2}},
$$




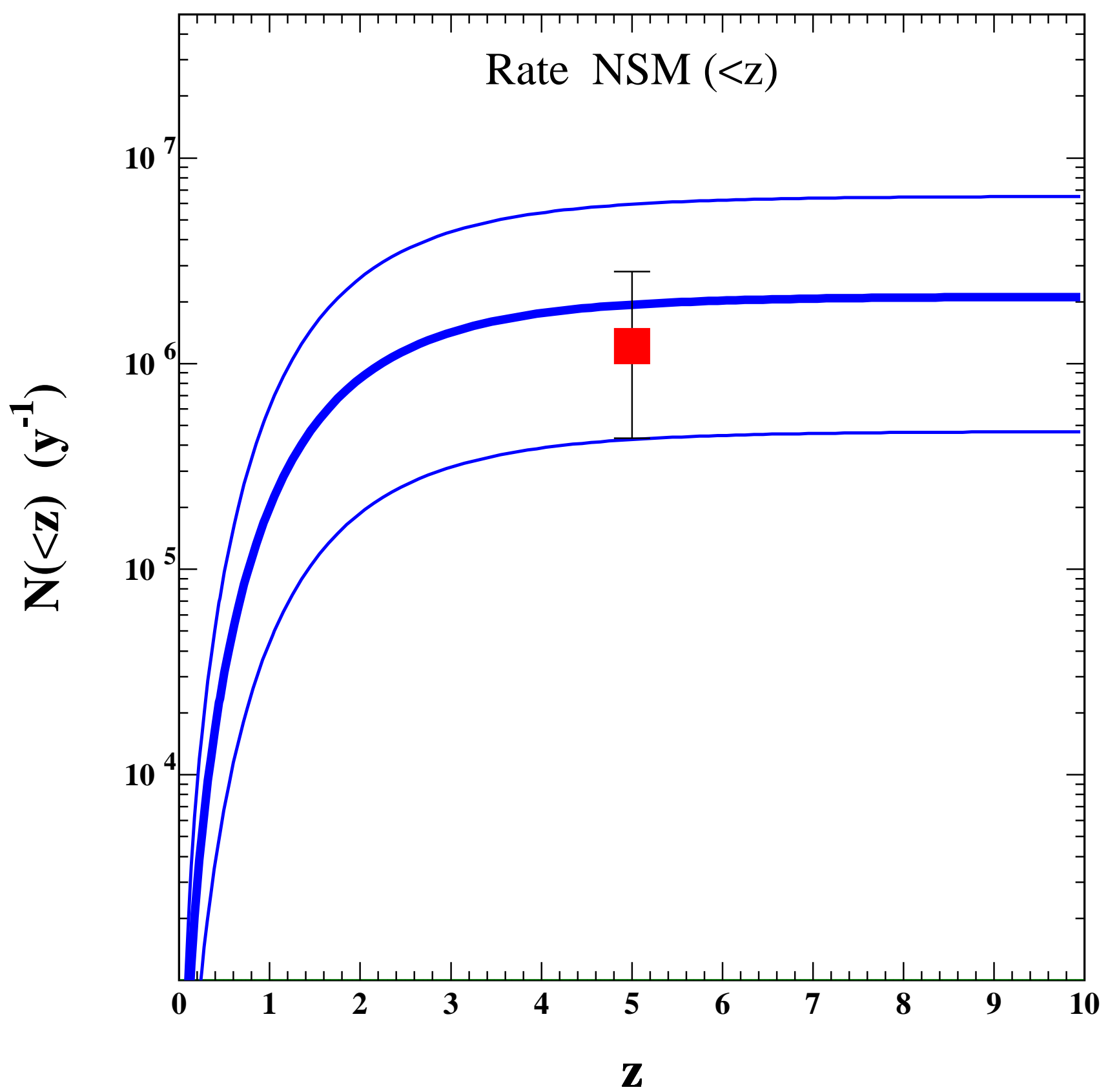

FIG. 2: The expected full sky rate of neutron stars merger (NSM) with redshift $\leq z$, as a function of $z$. The calculated rate is based on the standard cosmological model and the assumption that the NSM rate as a function of redshift $z$ is proportional to the observed star formation rate, SFR(z), as parametrized in eq.(12). The full and thin lines correspond to the estimated rate and its errors in a comoving $G p c^{3}$ volume reported in [13] by the Ligo-Virgo collaboration. The inserted point is the full sky rate estimated in [24], after subtracting the contribution of CDF-S XT1. 
where $E_{p}(t)$ is the peak energy at time $t$. For XRFs, i.e., far off axis GRBS, the exponential factor on the right hand side of eq.(13) can be neglected, which yields a full width at half maximum $\mathrm{FWHM} \approx 2 \Delta$, a rise time $R T \approx 0.59 \Delta$ from half peak to peak at $t=\Delta$, and a decay time $D T \approx 1.41 \Delta$ from peak to half peak. This pulse shape is in good agreement with that observed in resolved, well sampled, GRB pulses [17,27]. Figure 3 shows a comparison between eq.(13) and the measured light curve of CDF-S XT1. The best fit normalization, a pulse start up time $t_{0}=38.8 \mathrm{~s}$, and $\Delta=50.6 \mathrm{~s}$, yield $\chi^{2} / d o f=4.52 / 9=0.50$.

\section{CONCLUSIONS}

The observed light curve of CDF-S-XT2 recently discovered with the Chandra x-ray observatory [23] and the estimated full sky rate of such extragalactic fast x-ray transients support the conclusion that it was an early time nebular emission powered by a newly born millisecond pulsar with a strong magnetic field, $B \gtrsim 10^{14}$ Gauss in a neutron stars binary merger which produced SGRB beamed away from the direction of Earth $[21,23]$. The estimated strength of the dipole magnetic field of these newly born pulsars, from the afterglow which they power, depends on the assumption that their spin down is dominated by magnetic dipole radiation, which may or may not be true. The typical signature of orphan afterglows of SGRBs - a fast rise after burst followed by a short plateau phase of typically a few thousands of seconds, which turns into a fast temporal decline, may partially explain why such transients have not been found so far in searches of electromagnetic afterglows of SGRBs from the nearby binary neutron star merger candidates detected in gravitational wave by Ligo-Virgo in run O3 [39]. Fast extragalactic x-ray transients, such as CDF-S XT1 [22], which unlike CDF-S XT2 [23], do not have the "universal shape" of an early time pulsar powered afterglow of SGRB [19]. Its light curve has the universal shape of well resolved prompt emission pulses of LGRBs viewed far off axis, i.e. of X-ray flashes (XRFs), which were discovered in archival CDF data [40] long before CDF-S XT1 [22].

Acknowledgenents: We thank P. Jonker, Y. Q. Xue and an anonymous referee for useful comments. 


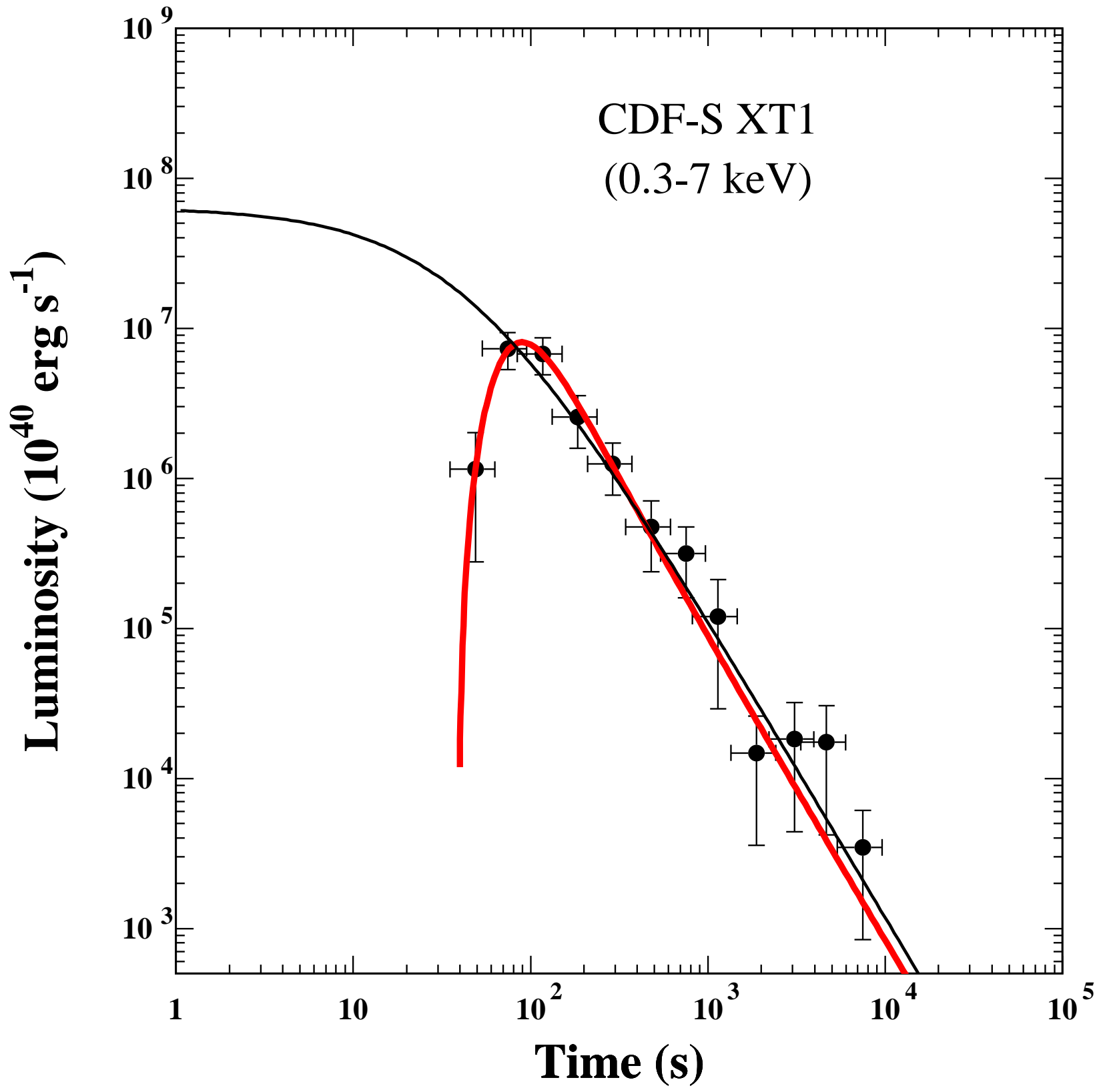

FIG. 3: Comparison between the observed pulse shape of CDF-S XT1 [22] in the 0.3-7 keV x-ray band and (a) the expected pulse shape of far off axis LGRB pulse as given by eq.(13) (thick red line) with the best fit parameters listed in the text, which yield $\chi^{2} / d f=0.50$, (b) the best fit universal shape of a nebular x-ray emission powered by the spin down of a newly born MSP (thin black line), as given by eq.(5). 
[1] S. I. Blinnikov, et al. 1984, Soviet Astron. Lett., 10, 177 (1984) arXiv:1808.05287.

[2] L. X. Li, B. Paczynski, ApJ. 507, L59 (1998) arXiv:astro-ph/9807272.

B. D. Metzger, e-print arXiv:1910.01617, and references therein (2019).

[3] J. Goodman, A. Dar, S. Nussinov, ApJ, 314 (1987) L7.

[4] C. A. Meegan, et al., Nature, 355, 143 (1992).

[5] J. Goodman, ApJ, 308, L47 (1986).

[6] N. Shaviv, A. Dar, ApJ, 447, 863 (1995). arXiv:astro-ph/9407039].

A. Dar, ApJ, 500, L93 (1998) arXiv:astro-ph 9709231.

[7] E. Costa, F. Frontera, J. Heise, et al., Nature, 387, 783 (1987) arXiv:astro-ph/9706065].

[8] J. van Paradijs, P. J. Groot, T, Galama, et al. Nature. 386, 686 (1997)

M. R. Metzger, S. G. Djorgovski, S. R. Kulkarni, et al. Nature, 387, 878 (1997)

D. A. Frail, S. R. Kulkarni, L. Nicastro, L., et al. Nature, 389, 261 (1997).

[9] A. Dar, ApJ, 500, L93, (1998) arXiv:astro-ph/9709231.

[10] R .A. M. J. Wijers, M. J. Rees, P. Meszaros, MNRAS, 288, L51 (1997) arXiv:astro-ph/9704153.

P. Meszaros, M. J. Rees, ApJ 476, 232 (1997) arXiv:astro-ph/9606043.

[11] T. Piran, Phys. Rept. 314, 575 (1999) arXiv:astro-ph/9810256.

[12] T. Piran, Phys. Rep. 333, 529 (2000) arXiv:astro-ph/9907392.

[13] B. P. Abbott, et al. [Ligo-Virgo Collaboration], PRL, 119, 161101 (2017) arXiv:1710.05832];

ApJ, 848, L12 (2017) arXiv:1710.05834]; ApJ, 851, L16 (2017) arXiv:1710.09320].

[14] A. Goldstein, et al., ApJ, 848, L14 (2017) arXiv:1710.0544.

[15] K. P. Mooley, et al., Nature 561, 355 (2018) arXiv:1806.09693.

[16] I. Arcavi et al., Nature, 551, 64 (2017) arXiv:1710.05843.

P. S. Cowperthwaite et al., ApJL, 848, L17 (2017) [arXiv:1710.02144].

M. R. Drout, A. L. Piro, OB. J. Shappee, et al., Science, 358, 1570 (2017) arXiv:1710.054431].

M. M. Kasliwal et al., et al., Science, 358, 1559 (2017) [arXiv:1710.05436].

M. Soares-Santos, et al., ApJL, 848, L16 (2017) arXiv:1710.05459.

B. D. Metzger, e-print arXiv:1910.01617, and references therein.

[17] S. Dado, A. Dar, Alvaro De Rújula, e-print arXiv:1712.09970 (2017). 
[18] E. Berger, W. Fong, R. Chornock, ApJ, 774, L23 (2013) arXiv:1306.3960].

N. R. Tanvir, et al., Nature, 500, 547 (2013) arXiv:1306.4971.

[19] S. Dado, A. Dar, PRD, 99, 123 (2019) arXiv:1807.08726].

[20] P. A. Evans et al., MNRAS, 397, 1177 (2009) arXiv:0812.3662.

P. A. Evans et al., A\&A, 469, 379 (2007) arXiv:0704.0128.

[21] S. Dado, A. Dar, Nuovo Cim. C41, 131 (2019) arXiv:1708.04603.

D. Xiao, B. B. Zhang, Z. G. Dai, ApJ 879, L7 (2019) [arXiv:1904.05480].

[22] F. E. Bauer, E. Treister, K. Schawinski, et al., 2017, MNRAS, 467, 4841 (2017) arXiv:1702.04422].

[23] Y. Q. Xue, et al., Nature, 568, 198 (2019) arXiv:1904.05368].

[24] G. Yang, W. N. Brandt, S. F. Zhu, F. E. Bauer, B. Luo, Y. Q. Xue, X. C. Zheng arXiv:1906.02793].

[25] H. Sun, et al., e-print arXiv:1908.01107 (2019).

[26] S. Dado \& A. Dar, e-print arXiv:1810.03514, and references therein.

[27] S. Dado \& A. Dar, e-print arXiv:1810.03514, and references therein.

[28] J. E. Rhoads, ApJ, 525, 737 (1999) arXiv:astro-ph/9903399.

[29] B. P. Abbott, et al. [Ligo-Virgo Collaboration], PRL, 119, 161101 (2017) arXiv:1710.05832.

[30] Other mechanisms by which newly born MSPs power GRBs and/or their afterglows were suggested, e.g., by

E. Blackman, I. Yi, ApJ, 498, L31 (1998) [arXiv:astro-ph/9802017].

Z. G. Dai and T. Lu, PRL, 81, 4301 (1998) arXiv:astro-ph/9810332.

Z. G. Dai and T. Lu, A\&A, 333, L87 (1998) arXiv:astro-ph/0602525.

B. D. Metzger, E. Quataert, T. A. Thompson, MNRAS, 385, 1455 (2008) arXiv:0712.1233.

A. Rowlinson, et al., MNRAS 409, 531 (2010) [arXiv:1007.2185].

B. D. Metzger, A. L. Piro, MNRAS, 439, 3916 (2014) arXiv:1311.1519].

B. P. Gompertz, P. T. O'Brien, G. A. Wynn, MNRAS, 438, 240 (2014) arXiv:1311.1505].

H. Lu, B. Zhang, W. Lei, Y. Li, P. D. Lasky, MNRAS, ApJ, 805, 89 (2015) arXiv:1501.02589.

S. Gibson, G. Wynn, B. Gompertz, P. O’Brien, MNRAS, 470, 4925 (2017) arXiv:1706.04802.

[31] J. H. Taylor, J. M. Weisberg, ApJ, 253,908 (1982).

[32] Originally, magnetars were defined to be pulsars powered by the decay of their ultrastrong magnetic field. Anomalous X-ray pulsars (AXPs) and soft gamma ray repeaters (SGRs), whose 
observed luminosity was found to exceed their loss of rotational energy [see, e.g., S. Mereghetti, AAR, 15, 225 (2008) arXiv:0804.0250], were the first discovered pulsars with a luminosity exceeding their spin down energy release. They were assumed to be powered by the decay of their assumed ultrastrong magnetic field. More recently, the name magnetars was extended to include millisecond pulsars (MSPs) whose spin down was assumed to be dominated by the emission of magnetic dipole radiation. See, e.g., [24].

[33] M. R. Drout, A. L. Piro, OB. J. Shappee, et al., Science. 358, 1570 (2017) arXiv:1710.054431].

[34] S. Dado \& A. Dar, ApJ, 785, 70 (2014) arXiv:1307.5556].

[35] N. Aghanim, et al., (Planck Collaboration), 2018 e-Print: arXiv:1807.06209.

[36] A. M. Hopkins, J. F. Beacom, ApJ, 651, 142 (2006) arXiv:astro-ph/0601463.

[37] N. A. Reddy, \& C. C. Steidel, ApJ, 692, 778 (2009) arXiv:0810.2788].

[38] S. Dado, A. Dar, A., De Rújula, A\&A, 422, 381 (2004) arXiv:astro-ph/0309294.

S. Dado, A. Dar, ApJ, 884 L44, (2019) arXiv:1908.05116] and references therein.

[39] LIGO-Virgo Gravitational-Wave Candidate Event Database, https://gracedb.ligo.org/superevents/public/O3/

[40] P. G. Jonker, et al., ApJ, 779, 14 (2013) arXiv:1310.7238].

A. Glennie, P. G. Jonker, R. P. Fender, T. Nagayama, M. L. Pretorius, MNRAS, 450, 3765 (2015) arXiv:1504.03720]. 